\title{
Noncovalent Complexes Between DNA and Basic Polypeptides or Polyamines by MALDI-TOF
}

\author{
Peran Terrier, Jeanine Tortajada, Grégoire Zin, and William Buchmann \\ Laboratoire Analyse et Modélisation pour la Biologie et l'Environnement, Université d'Evry-Val d'Essonne, \\ Evry, France
}

MALDI-MS was evaluated as a method for the study of noncovalent complexes involving DNA oligonucleotides and various polybasic compounds (basic polypeptides and polyamines). Complexes involving single-stranded DNA were successfully detected using DHAP matrix in the presence of an ammonium salt. Control experiments confirmed that the interactions involved basic sites of the polybasic compounds and that the complexes were not formed in the gas phase but were pre-existing in the matrix crystals. Moreover, the pre-existence in solution was probed by isothermal titration calorimetry at concentration and ionic strength similar to those used for mass spectrometry. Spectra showed no important difference between negative and positive ion modes. The influence of nature and size of DNA and polybasic compound on the relative intensities and stoichiometries of the complexes was investigated. Despite the fact that relative intensities can be affected by ionization yields and the gas-phase stabilities of the different species, numerous trends observed in the MALDI study were consistent with the expected in-solution behaviors. Experimental conditions related to sample preparation were investigated also. Complex abundance generally decreased when increasing the ammonium acetate concentration. It was dramatically decreased when using ATT instead of DHAP. Penta-L-arginine is an exception to these observations. Lastly, in the case of complexes involving DNA duplex, the ATT matrix was shown to favor the observation of specific DNA duplex but not that of its complex with polybasic compounds. Inversely, DHAP was appropriate for the conservation of DNA-polybasic compound interaction but not for the transfer of intact duplex. (J Am Soc Mass Spectrom 2007, 18, 1977-1989) (c) 2007 American Society for Mass Spectrometry

$\mathrm{B}$ asic polypeptides (polylysines, polyarginines, polyhistidines), synthetic polyamines, and derivatives arouse a great interest within the context of gene therapy $[1,2]$. They are possible candidates as vectors to introduce deoxyribonucleic acid (DNA) into a cell. Indeed, their positive charges allow ionic interactions with the negative charges of both the DNA and the cellular membrane. However, such vectors are still relatively inefficient. A better understanding of the interactions between the vector and the DNA could permit further optimization for more efficient gene delivery.

With the introduction and the development of soft ionization methods such as electrospray ionization (ESI) [3] and matrix-assisted laser desorption/ionization (MALDI) [4, 5], mass spectrometry has become a powerful tool not only for the observation of intact biomolecules but also for their noncovalent assemblies. ESI-MS is generally recognized as the method of choice for the

Address reprint requests to Dr. William Buchmann, Laboratoire Analyse et Modélisation pour la Biologie et l'Environnement, Université d'Evry-Val d'Essonne, CNRS UMR 8587, Bât. Maupertuis, Bd. F. Mitterrand, 91025 Evry cédex, France. E-mail: william.buchmann@univ-evry.fr study of noncovalent complexes [6]. However, to our knowledge, this technique has not been used to survey complexes between DNA and polybasic compound until a recent report from our group [7]. Nevertheless, several works by ESI-MS focused on related systems in which two peptides formed a noncovalent complex by ionic bonds [8-11].

MALDI-MS is generally less frequently applied to the study of noncovalent complexes than ESI-MS. Indeed, on top of the relative harshness of the laser desorption/ionization process, sample preparation for this technique can be considered as critical for the survival of the complexes. The analyte has to be embedded within the matrix crystals. As the matrix is often an acidic organic compound, both the $\mathrm{pH}$ and the nature of the solvent required to dissolve the complex can induce its dissociation (leading to false negatives). Another problem, shared with the ESI technique, is the possible formation in the gas phase of aggregates that do not reflect the in-solution species (leading to false positives). However, several works have shown that great care taken in sample preparation allowed the transfer of intact complexes from the solution to the gas phase via matrix crystals [12]. 
Noncovalent complexes involving polybasic peptides and DNA have been detected by MALDI-TOF [13-18]. The first observation of such complexes was reported by Juhasz and Biemann [13]: highly acidic compounds (such as DNA) were detected as noncovalent complexes with a peptide or a small protein rich in arginine, lysine and/or histidine. Subsequently, Vertes et al. [14] observed interactions between single stranded DNA and basic dipeptides or small proteins. In these works, acidic matrixes such as 3,5-dimethoxy-4hydroxycinnamic acid (sinapinic acid) or 2,5-dihydroxybenzoic acid (DHB) were mainly used. Vertes et al. [14] showed that basic peptides were bound to DNA by ionic interactions between negatively charged DNA phosphates and protonated peptide residues. However, due to the acidic $\mathrm{pH}$ of the matrix solution, adenine, cytosine, and guanidine were protonated and formed an intramolecular ionic bond with a nearby phosphate, preventing the binding to basic residues of peptides. Only phosphates located near the neutral thymines could form an ionic bond with a protonated site of a peptide in this case. On the other hand, with 6-aza-2-thiothymine (ATT, a nonacidic matrix) in ammonium citrate solution ( $\mathrm{pH}$ 6.5), interactions with other bases than thymine could be observed. The same year, Lecchi and Pannell [19] showed that ATT with ammonium citrate permitted the observation of intact DNA duplexes by MALDI-MS. Subsequently, Woods et al. [15] used this matrix to observe complexes between various basic peptides and single/ double stranded DNA. ATT (at times in the presence of ammonium citrate) has been successfully used also to observe other complexes involving DNA and derivatives of guanidinium (which is the basic site of the arginine residues) [16] or peptides [17, 18], or between two peptides bound by ionic bonds [8, 20-22]. In all these reports focusing on acid/base interactions, guanidinium seemed to play an important role as the basic site. Complexes involving only lysine or histidine residues are seldom observed. This can be explained by a greater stability of the arginine's bond with phosphate (or carboxylate) group in solution or in the gas phase due to its structure. Zehl and Allmaier [23] used peptide-peptide complexes involving patterns described by Woods et al. [20] as leading to specific solution-phase complexes (Arg-Arg or Arg-Lys$\left.\mathrm{Arg} /(\mathrm{Glu})_{5}\right)$ to try a wide range of matrixes and sample preparations. A majority of these matrixes gave poor results (particularly the acidic ones). On the other hand, it has been shown that ATT and 2,4,6-trihydroxyacetophenone (THAP) used with an ammonium buffer allowed the observation of intense and specific complexes. It was also found that 2,6-dihydroxyacetophenone (DHAP) allowed the observation of complexes. However, in this case, the intensities of the specific complexes were inferior to $5 \%$ and nonspecific complexes were detected.

Observations of complexes involving DNA and polyamine by mass spectrometry have been reported only in the case where spermine was used as a matrix additive for DNA analysis by MALDI-TOF [24-27]. Protonated sites of the polyamine were expected to bind with DNA phosphates in solution to displace alkaline cations [24-26] and stabilize duplexes [27]. Ideally, polyamine protons were transferred to DNA phosphates during sample crystallization or desorption/ionization process and DNA free of alkaline cation and polyamine was observed. But under some matrix conditions, unwanted polyamine-DNA complexes were detected.

In this work, MALDI-MS was evaluated as a method for the study of noncovalent complexes involving DNA and basic polypeptides (penta-L-arginine, penta-Llysine) or polyamines (polypropylenimine dendrimers, linear and branched polyethylenimines). It was expected that DNA and polybasic compounds mainly interact via ionic bonds between phosphates and protonated amino sites. In contrast to previously reported studies, the majority of the compounds we used contained no arginine and many of them were polydisperse. The influence of the nature and the size of the polybasic compound was studied, as well as the composition, the size and the form (single-stranded or duplex) of the DNA. The influence of experimental conditions (nature of the matrix, ionic strength) on the spectra of complexes was explored too. Moreover, for some polybasic compounds, microcalorimetry experiments have been carried out under the same conditions (same buffer, similar concentration range) to compare results obtained using MALDI mass spectrometry with those deduced from an "in-solution" technique.

The expected advantages of MALDI-MS over ESI-MS were the increased rapidity and simplicity. Moreover, this technique produces mainly singly charged ions, so spectra are easier to interpret. These features, making MALDI-MS the most appropriate method for the analysis of synthetic polymers, appeared promising for the study of noncovalent complexes involving synthetic polymers.

\section{Experimental}

\section{Materials}

Oligodeoxyribonucleotides, polypeptides, polyamines, and poly(ethylene glycol) used in this study are listed in Table 1. All oligodeoxyribonucleotides and penta-Larginine were purchased from Eurogentec (Seraing, Belgium). Penta-L-lysine, penta-L-alanine, poly(ethylene glycol) and all polyamines (described in Figure 1) were purchased from Sigma-Aldrich (Lyon, France). The latter company also supplied ammonium acetate, ammonium citrate, $2^{\prime}$, $6^{\prime}$-dihydroxyacetophenone (DHAP) and 6-aza-2-thiothymine (ATT). All compounds were used as received.

\section{Mass Spectrometry}

DNA duplexes were prepared by mixing two complementary strands at $10^{-3} \mathrm{M}$ each in aqueous ammonium acetate $(100 \mathrm{mM})$. Then, the solution was annealed at $90^{\circ} \mathrm{C}$ for $5 \mathrm{~min}$, cooled for a further $3 \mathrm{~h}$ to room 
Table 1. Oligodeoxyribonucleotides, polypeptides, polyamines, and polyether used in this study. The two values (X/Y) of average molecular masses (and resulting number of basic sites) of polyethylenimines are those measured in our laboratory by ESI-MS (X) and given by the supplier $(\mathrm{Y})$

\begin{tabular}{|c|c|c|c|}
\hline \multicolumn{4}{|c|}{ Oligodeoxyribonucleotides } \\
\hline DNAi & 5'-AAGCCCGCCCAA-3' & $\mathrm{M}=3584.4 \mathrm{Da}$ & 11 phosphate groups \\
\hline DNAj & 5'-TTGGGCGGGCTT-3' & $\mathrm{M}=3708.4 \mathrm{Da}$ & 11 phosphate groups \\
\hline DNAk & 5'-AAAAGCCCGCCCGCCCGCCCAAAA-3' & $\mathrm{M}=7230.7 \mathrm{Da}$ & 23 phosphate groups \\
\hline \multicolumn{4}{|l|}{ Polypeptides } \\
\hline R5 & penta-L-arginine & $\mathrm{M}=798.9 \mathrm{Da}$ & 5 lysine residues \\
\hline $\mathrm{K} 5$ & penta-L-lysine & $\mathrm{M}=658.9 \mathrm{Da}$ & 5 arginine residues \\
\hline A5 & penta-L-alanine & $\mathrm{M}=373.4 \mathrm{Da}$ & no basic residue \\
\hline \multicolumn{4}{|l|}{ Polyamines } \\
\hline DAB4 & polypropylenimine tetraamine dendrimer & $\mathrm{M}=316.5 \mathrm{Da}$ & 6 amino groups \\
\hline DAB8 & polypropylenimine octaamine dendrimer & $\mathrm{M}=773.3 \mathrm{Da}$ & 14 amino groups \\
\hline DAB16 & polypropylenimine hexadecaamine dendrimer & $\mathrm{M}=1686.8 \mathrm{Da}$ & 30 amino groups \\
\hline LPEI400 & linear polyethylenimine & $\mathrm{Mn} \sim 310 / 400 \mathrm{Da}$ & $\sim 7 / 9$ amino groups \\
\hline BPEI600 & branched polyethylenimine & $\mathrm{Mn} \sim 550 / 600 \mathrm{Da}$ & $\sim 12 / 14$ amino groups \\
\hline BPEI1200 & branched polyethylenimine & $\mathrm{Mn} \sim 850 / 1200 \mathrm{Da}$ & $\sim 19 / 28$ amino groups \\
\hline BPEI1800 & branched polyethylenimine & $\mathrm{Mn} \sim 1150 / 1800 \mathrm{Da}$ & $\sim$ 26/41 amino groups \\
\hline \multicolumn{4}{|l|}{ Polyether } \\
\hline PEG1000 & poly(ethylene glycol) & $\mathrm{Mn} \sim 1000 \mathrm{Da}$ & no basic group \\
\hline
\end{tabular}

temperature and diluted 50 times in $100 \mathrm{mM}$ ammonium acetate before the preparation of the complex.

Typically, equimolar solutions containing $10 \mu \mathrm{M}$ each of single-stranded (or duplex) DNA and polybasic compound were prepared in aqueous ammonium acetate solution (100 mM, pH 6.5). For polydisperse compounds, concentrations were calculated taking into consideration the average molecular mass provided by the supplier. The solutions were kept at room-temperature for $15 \mathrm{~min}$ before analysis.

MALDI-TOF experiments were carried out using a Perseptive Biosystems Voyager-DE Pro STR mass spectrometer (Applied Biosystems/MDS SCIEX, Foster City, CA) equipped with a nitrogen laser (wavelength $=337 \mathrm{~nm}$, frequency $=20 \mathrm{~Hz}$; fluence was set just above the desorption-ionization threshold). The mass spectrometer was operating in linear mode. According to the acceleration mode (positive or negative), acceleration voltage was + or $-25 \mathrm{kV}$. Grid voltage was $93 \%$ and extraction delay was $470 \mathrm{~ns} .100 \mathrm{mM}$ matrix (DHAP or ATT) and $10 \mathrm{mM}$ ammonium citrate solutions were prepared in 50/50 (vol/vol) water/acetonitrile. Just before analysis, equal volumes of matrix solution and complex aqueous solution $(10 \mu \mathrm{M})$ were mixed, spotted onto MALDI plate and allowed to dry at room temperature.

\section{Isothermal Titration Calorimetry}

Isothermal titration calorimetry experiments (ITC) were performed using a VP-ITC isothermal titration calorimeter (Microcal, Northampton, MA). Experiments were performed at $20^{\circ} \mathrm{C}$. Polybasic compounds and DNA were dissolved in aqueous solution containing $100 \mathrm{mM}$ acetate ammonium. Typically, 28 injections of $10 \mu \mathrm{L}$ of a $1 \mathrm{mM}$ polybasic compound solution were added from a computer-controlled microsyringe at intervals of $180 \mathrm{~s}$ into a $1.430 \mathrm{~mL}$ cell containing a $20 \mu \mathrm{M}$ DNA solution.
For each complex, others instrumental parameters (polybasic compound concentration, number, and volume of the injections) were tried to confirm the results. Reference experiments were performed by injecting the polybasic compound into a cell containing only the acetate ammonium. Reference values were subtracted from the sample values solution. A theoretical titration curve (calculated with an on-site model) was fitted to the experimental data using the Origin(r) 7SR2 software (Microcal).

\section{Results and Discussion}

\section{Isothermal Titration Calorimetry}

In this study, noncovalent complexes involving DNA and basic polypeptides (penta-L-lysine and penta-Larginine) or polyamines (polypropylenimine dendrimers and polyethylenimines, see Table 1 and Figure 1) were studied. Preliminary isothermal titration calorimetry (ITC) experiments were carried out to ensure that the solution conditions used for mass spectrometry (10
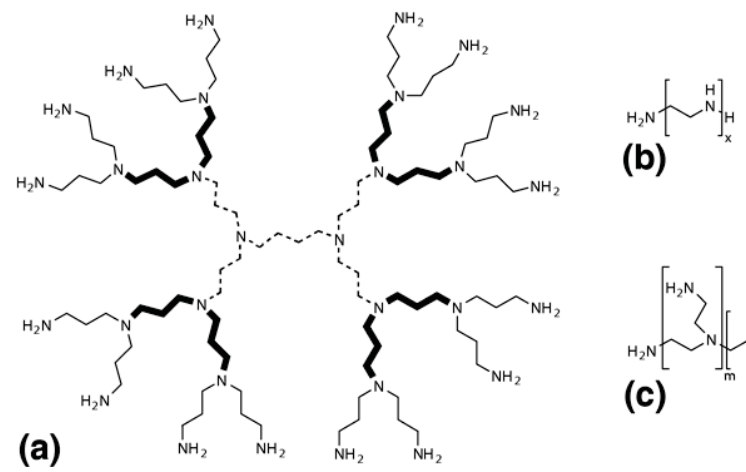

(b)

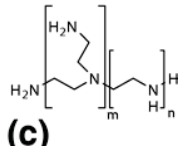

(c)

Figure 1. (a) Polypropylenimine dendrimers. Dashed: DAB4, dashed + bold: DAB8, all: DAB16. (b) Linear polyethylenimine. (c): Branched polyethylenimine. 
$\mu \mathrm{M}$ each component in $100 \mathrm{mM}$ ammonium acetate aqueous solution) permitted the formation of complexes. For these ITC experiments, we chose complexes involving a 12-mer oligonucleotide (DNAi) and polypropylenimine dendrimers (DAB4, DAB8, and DAB16) as models. Indeed, contrary to the polyethylenimines, polypropylenimine dendrimers are monodisperse, making easier the measurement by ITC.

Unfortunately, the (DNAi + DAB4) interaction was too weak to be observed by calorimetry. For the (DNAi + DAB8) interaction, the dissociation constant $\left(\mathrm{K}_{\mathrm{D}}\right)$ was found between $1 \times 10^{-6} \mathrm{M}$ and $2.3 \times 10^{-6} \mathrm{M}$. For (DNAi + DAB16), an interaction has been observed, but no quantitative value could be unambiguously determined, due to precipitation phenomenons.

Nevertheless, these preliminary experiments confirmed that the conditions used in this study were compatible with the existence of the noncovalent complexes in solution. The difficulties encountered with microcalorimetry supported the need for developing new mass spectrometric methods for this kind of application.

\section{Observation of Noncovalent Complexes}

A noncovalent complex was detected by MALDI-TOF for each combination of a DNA (DNAi, DNAj, DNAk) and a polybasic compound (polybasic peptides and polyamines, see Table 1 ). These experiments were carried out by using DHAP as the matrix in the presence of ammonium citrate. Both negative and positive ion modes were successful.

For the purpose of discussion, we have chosen to show as examples the spectra of complexes involving the 12-mer oligonucleotide DNAi and three polybasic compounds: penta-L-lysine (K5), polypropylenimine octa-amine dendrimer (DAB8), and branched polyethylenimine (BPEI1200) in both positive and negative ion modes (Figure 2b, c, d, f, g, and h). Spectra of DNAi alone are also given as references (Figure $2 \mathrm{a}$ and e). All observed species were charged by deprotonation(s) or protonation(s). Since matrix peaks and fragment peaks were sometimes numerous and abundant in the lower mass region, spectra are displayed from $m / z 1500$. However, peaks corresponding to free polybasic compounds could be detected among these numerous unwanted peaks in the positive ion mode (only K5 was detected in the negative ion mode as well). In all the spectra, free DNAi was detected under its singly and doubly charged form. Although DNAi is not selfcomplementary, its dimer was observed too (in a small amount). In each case, complexes involving DNAi and the polybasic compound with various stoichiometries were detected. The most intense complex always corresponded to a (1:1) stoichiometry and was present under its singly and doubly charged forms. In the case of the polydisperse BPEI1200 (Figure 2d and h), the complex was detected as a distribution of peaks separated by 43 atomic mass units, the mass of the ethylenimine repeat unit. Each peak corresponds to a complex involving one DNAi and one polyethylenimine chain with different degrees of polymerization (i.e., a different number of repeat units). Moreover, DNAi:polybasic compound complexes with (2:1) and (2:2) stoichiometries could be detected.

\section{Control Experiments}

Formation of aggregates in the gas-phase during MALDI process is a well-known phenomenon [28]. So, when studying noncovalent complexes between DNA and polybasic compounds, great care must be taken to be sure that observed complexes pre-exist in solution and are not formed in the gas phase. Although our experimental conditions include a relatively low analyte/matrix molar ratio $\left(10^{-4}\right)$, theoretically preventing the observation of non-relevant complexes, we carried out several control experiments. For these experiments (DNAi + DAB8) was chosen as a model.

First, we employed particular conditions for which the complex could not pre-exist in the MALDI deposit and hence (if observed) could only be formed in the gas phase. In this way, we hoped to determine if gas-phase association of the two components could occur under our experimental conditions. We used a special MALDI plate including an insert holder and a removable insert (see scheme on Figure 3). DNAi and DAB8 were separately spotted in the presence of the matrix (DHAP) on the two separated parts (one deposit on the insert holder and the other on the removable insert). Then, the dried deposits were brought together by replacing the removable insert into the insert holder. The spectra were recorded in positive ion mode with laser shots straddling both joined deposits. This experimental procedure enables the placement of the two components under the same laser shot without the possible complex formation inside the deposit. Under these conditions, free DAB8 and free DNAi were observed as singly protonated species but absolutely no complex (Figure $3 a)$, indicating that no complex was formed in the gas phase. On the contrary, when an incubated mixture of equimolar DAB8 and DNAi was spotted straddling both joined parts of the MALDI plate, and the spectrum was recorded with laser shots on the junction (Figure $3 b)$, an intense complex was detected on top of the free components. In a similar experiment reported by Bogan and Agnes [29], (polyethyleneglycol + lithium) noncovalent complexes were detected, indicating these conditions allowed the observation of gas-phase aggregates. However, the extent of plume overlap is not well-known and the lateral mixing of the components in the plume could be insufficient. To leave no doubt about the possible gas-phase aggregation between DNA and the polybasic compound within our experimental conditions, a second experiment was carried out. This experiment was previously described by Friess and Zenobi [30]. Two solutions were prepared separately, one containing DAB8 and DHAP, the other 


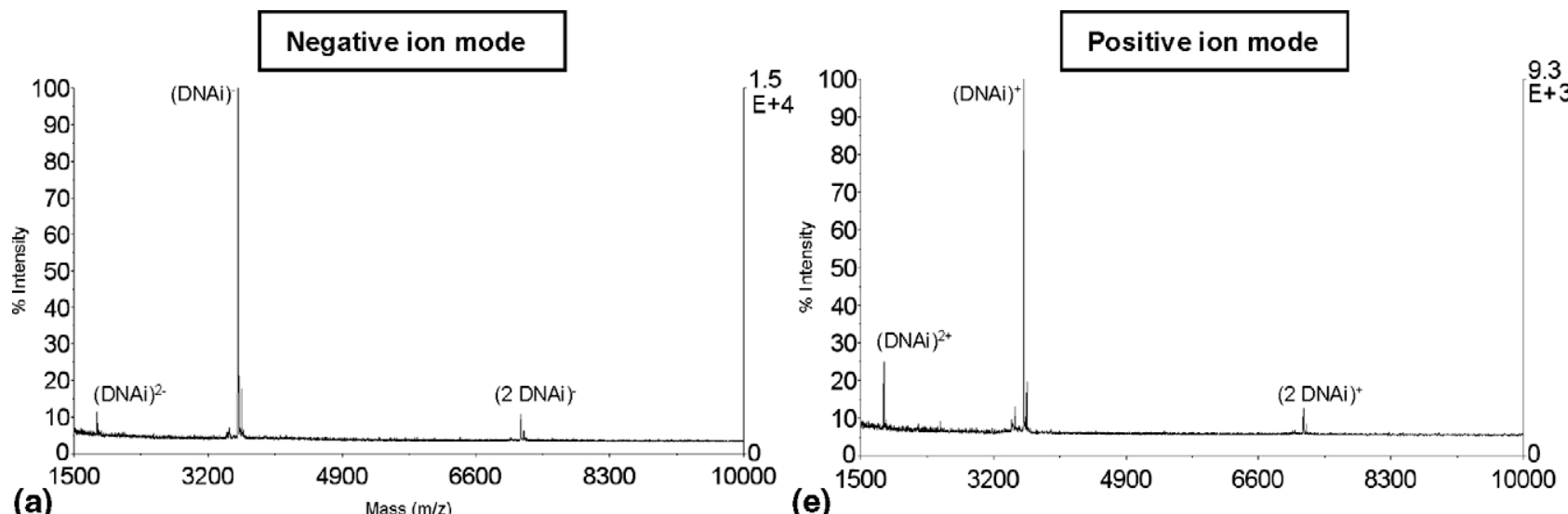

(a) $\operatorname{Mass}(\mathrm{m} / \mathrm{z})$

(e)
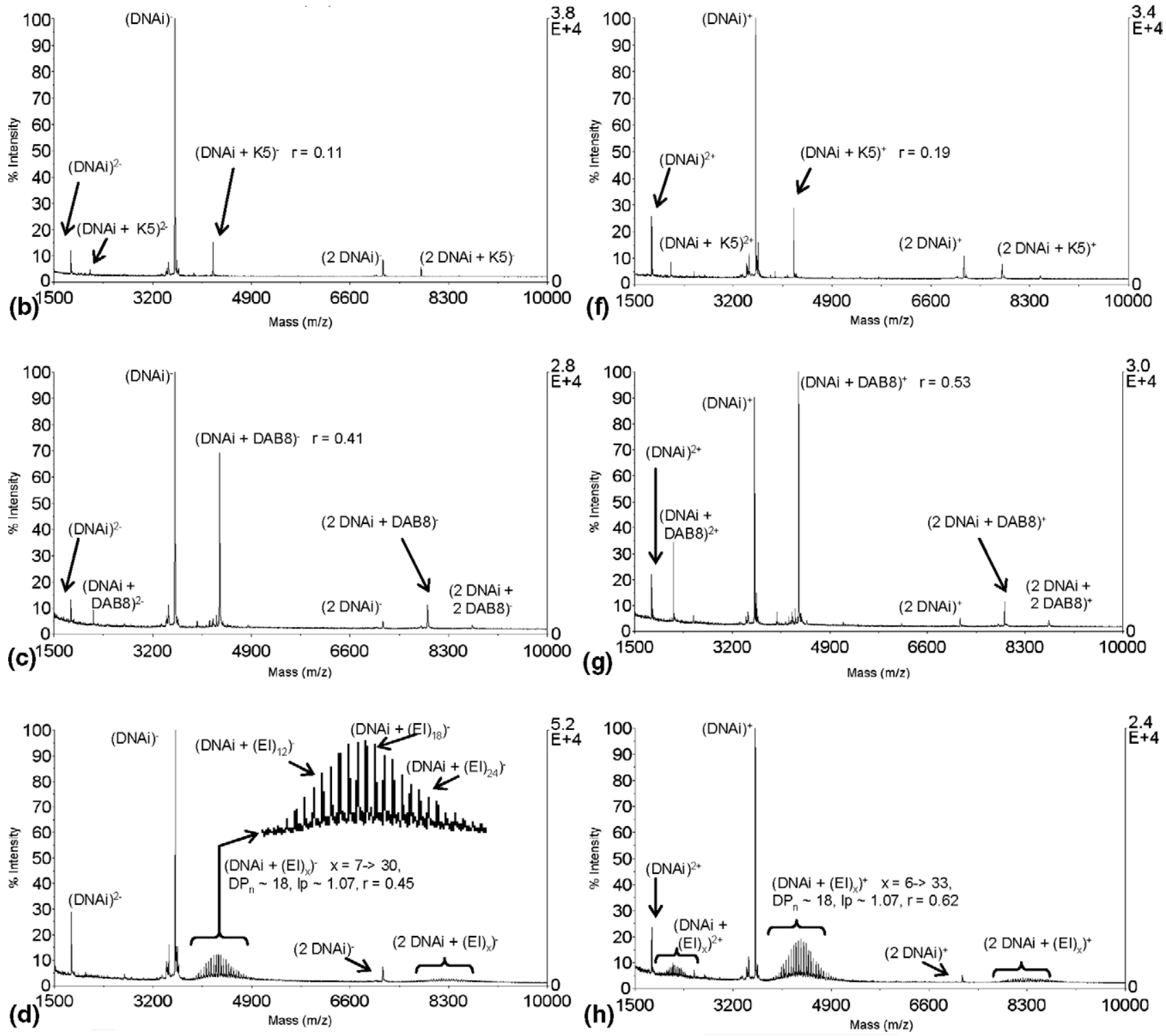

Figure 2. MALDI-TOF spectra of oligonucleotide DNAi alone (a) and (e), in the presence of K5 (b) and (f), in the presence of DAB8 (c) and (g), in the presence of BPEI1200 (d) and (h); (a), (b), (c), and (d) are recorded in the negative ion mode; (e), (f), (g), and (h) are recorded in the positive ion mode. [Ammonium acetate] $=100 \mathrm{mM}$. Matrix: DHAP/ammonium citrate. All charges are the result of deprotonation(s) or protonation(s). 


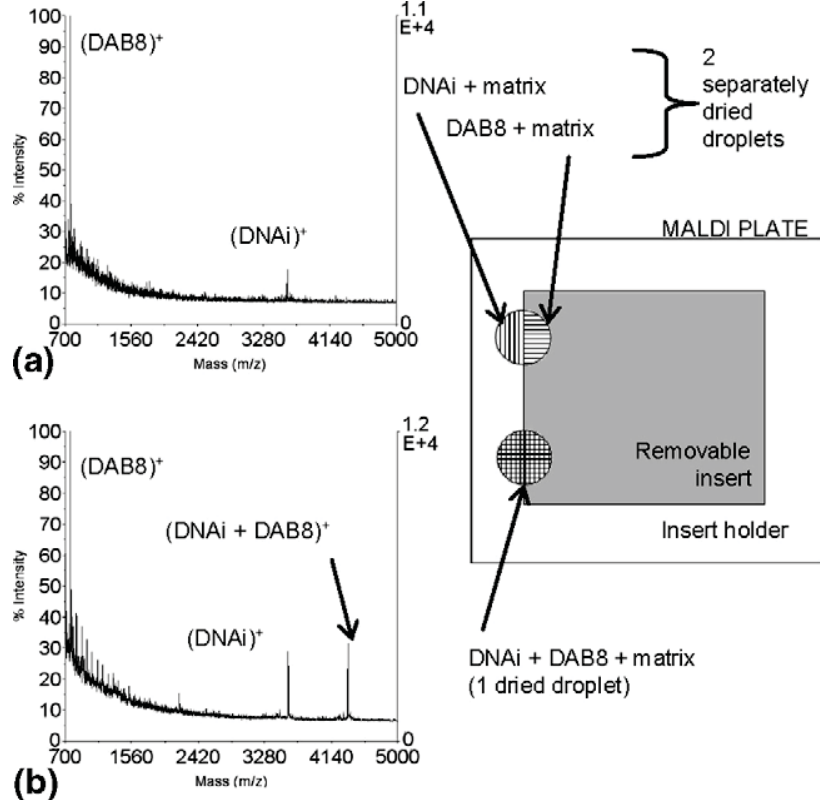

Figure 3. Positive ion mode MALDI-TOF spectra of DNAi in the presence of DAB8. (a) DAB8 and DNAi were separately dried with matrix on two separated parts of a special MALDI plate, and then both parts were joined. (b) A mixture of DNAi, DAB8, and matrix was spotted straddling both joined part. Both spectra were recorded with laser shots straddling both joined parts of MALDI plate. [Ammonium acetate] $=100 \mathrm{mM}$. Matrix: DHAP/ammonium citrate. All charges are the result of deprotonation(s) or protonation(s).

containing DNAi and DHAP, just as for two classical MALDI deposits. Then, these solutions were dried (using a rotative evaporator) and the resulting powders were mixed together. The mixture was then attached to the MALDI plate using electrically conductive doublesided adhesive tape. As previously, we finally obtained a deposit containing the two components but no complex. As shown in Figure $4 a$, free components were abundantly detected but no complex. After the deposition of a solvent droplet on the deposit, we were unfortunately not able to obtain a complex again due to solubilization difficulties. However, when a solution mixture of the two components (DNAi and DAB8) in the presence of matrix was spotted onto the adhesive tape (Figure $4 \mathrm{~b}$ ), the noncovalent complex was abundantly detected.

The results of these two experiments tend to show that the (DNAi + DAB8) complex was not formed in the gas phase but was already present in the deposit and survived the MALDI process. However the relative proportions of free components directly coming from the deposit over those coming from gas-phase decomposition of the complex are not known.

In the last control experiment (Figure 5), spectra of mixtures (DNAi + penta-L-alanine noted A5) and (DNAi + poly(ethylene glycol) noted PEG) were recorded under the same experimental and instrumental conditions as those used to record the spectra shown Figure 2. Unlike basic polypeptide and polyamine, A5 and PEG are neutral in solution and are not expected to interact with DNA in solution. In both cases, the MALDI-TOF spectrum showed free DNA but absolutely no complex (free PEG is detected, but not free A5, which was out of the mass range). This experiment confirmed that no gas-phase aggregation occurs between DNA and a neutral compound and that DNA interacts with polybasic compounds through their basic groups. This interaction is assumed to be ionic between negatively charged phosphates and protonated amino groups.

It is more difficult to probe the origin of the DNAi dimer. However, as DNAi is not self-complementary, it is probable that a part of the DNA auto-association occurred during the MALDI process. (2:1) and (2:2) complexes could result from similar association between DNA and (DNA + polybasic compound) during the MALDI process. They also could pre-exist in solution. Given the small abundance of the corresponding signals, no supplementary experiment was attempted.

\section{Negative Versus Positive Ion Mode}

To roughly quantify the apparent abundance of the complexes, the $r$ ratio of the complex signals over those of the complexed plus the non-complexed DNA was calculated for each singly charged complex involving one DNA molecule; $r$ value was displayed on each spectrum (Figure 2). Generally, for the doubly-charged species, the $r$ ratio is the same as for the singly charged species. Therefore, it was neither indicated nor discussed. Moreover, complexes involving 2 DNAs were not taken into consideration for the calculation of this ratio, since corresponding signals were sometimes not good enough to be correctly integrated. For the (DNAi + BPEI1200) complex, the sum of the areas of each complex peak was considered.

Strikingly, spectra of each mixture were similar in both negative and positive ion mode: detected species

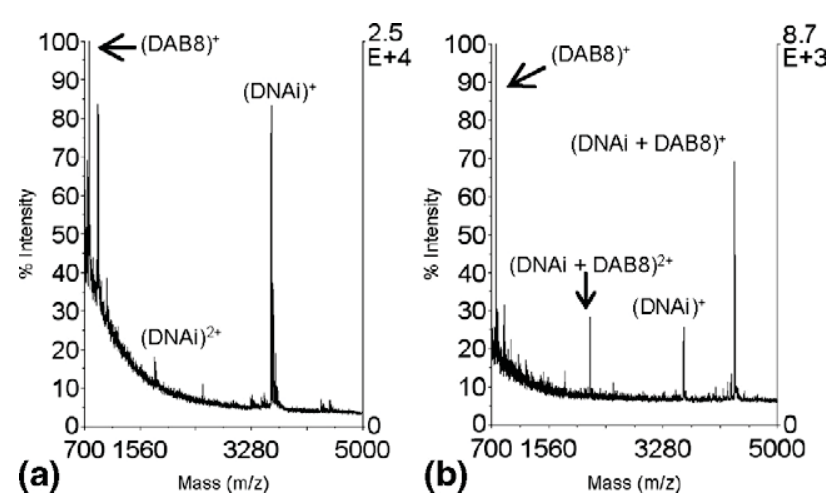

Figure 4. Positive ion mode MALDI-TOF spectra of DNAi in the presence of DAB8. (a) A powder mixture of DNAi, DAB8 and matrix was deposed on the MALDI plate owing to an electrically conductive double-sided adhesive tape. (b) A solution mixture of DNAi, DAB8 and matrix was deposed on the adhesive tape. [Ammonium acetate] $=100 \mathrm{mM}$. Matrix: DHAP/ammonium citrate. All charges are the result of deprotonation(s) or protonation(s). 

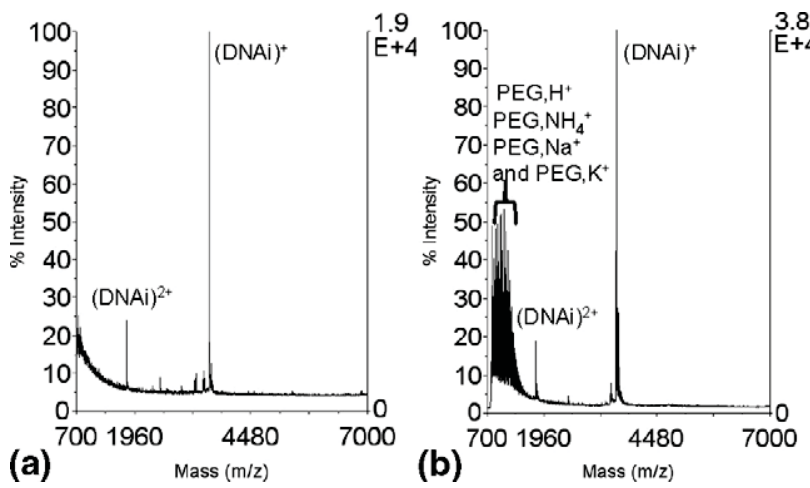

Figure 5. Positive ion mode MALDI-TOF spectra of DNAi in the presence of A5 (a) and PEG (b). [Ammonium acetate] $=100 \mathrm{mM}$. Matrix: DHAP/ammonium citrate. All charges are the result of deprotonation(s) or protonation(s).

were the same and no important differences in relative intensities were observed. However, the relative intensity of the complex was in each case slightly higher in the positive ion mode than in the negative ion mode. It is noteworthy that our conditions involve a neutral $\mathrm{pH}$ and a neutral matrix. Consequently, (DNAi + K5) complex has a large excess of negative charges in solution (DNAi has 11 phosphate groups and K5 has only 6 amino groups). This excess is at least decreased (if not reversed) in the case of (DNAi + DAB8) (11 phosphate groups versus 14 amino groups) and all the more in the case of (DNAi + BPEI1200) (11 phosphate groups versus 28 amino groups). We could have expected the in-solution negatively charged (DNAi + K5) complex to be favored in the negative ion mode (over the positive ion mode) and the (DNAi + BPEI1200) complex to be more favoured in the positive ion mode. The result suggests that, whatever the charge ratio and the nature of the polybasic compound, proton transfers between the complex and ammonium acetate (within the matrix or during the $470 \mathrm{~ns}$ extraction delay) occur efficiently in each case and lower the charge state of the complex, leaving either one positive or one negative charge without any important preference. The positive charge is likely to be located on one of the amino groups of the polybasic compound or on a DNA base, whereas the negative charge should be located on one of the DNA phosphate groups or on the terminal carboxylic acid of K5 (carboxylate).

Assuming that the peak intensities reflect the insolution abundances, theoretical $r$ ratio can be easily deduced from $K_{\mathrm{D}}$ measured by ITC with (DNAi + DAB8). With a $K_{D}$ value of $1 \times 10^{-6} \mathrm{M}, r$ ratio was found to be equal to 0.73 or 0.64 (depending on the considered initial concentration: that of the aqueous solution or that of the mixture with the matrix). For a $K_{D}$ of $2.3 \times$ $10^{-6} \mathrm{M}$, theoretical $r$ becomes equal to 0.62 or 0.51 . Experimental values are $r=0.41$ in the negative mode and $r=0.53$ in the positive mode. There is a relatively good agreement between theoretical and experimental values. The positive mode seemed to be more representative of the solution concentrations.
On the assumption that free DNA came directly from the deposit (and was not produced by gas-phase dissociation of the complex), the slightly greater $r$ ratios in the positive ion mode can be due to a lower ionization yield of the free DNA in the positive ion mode than in the negative ion mode, while complex transfer rate would be more independent of the ion mode. Another explanation could be that a part of the observed free components came from in-source gas-phase dissociation of the complex and that the positively charged complexes were more stable than the negatively charged ones.

The average degree of polymerization $\left(\mathrm{DP}_{n}\right)$ and the index of polydispersity $\left(\mathrm{I}_{\mathrm{p}}\right)$ can be calculated for BPEI1200 bound to DNA. In both negative and positive ion modes, these values are the same: $\mathrm{DP}_{n} \sim 18$ and $\mathrm{I}_{\mathrm{p}}$ $\sim 1.07$. The $\mathrm{DP}_{n}$ is inferior to the supplier data $\left(\mathrm{DP}_{n} \sim\right.$ 28) but relatively close to that measured for the free polymer in our laboratory using ESI-MS $\left(\mathrm{DP}_{n} \sim 19\right)$. In the same manner, $I_{p}$ of bound BPEI1200 is close to that of free BPEI1200 measured using ESI-MS ( $\left.\mathrm{I}_{\mathrm{p}} \sim 1.07\right)$. No $I_{p}$ value was given by the supplier. The fact that the observed $\mathrm{DP}_{n}$ are the same in both the negative and positive ion modes confirmed that positively and negatively charged species are formed without preference whatever the charge ratio in solution.
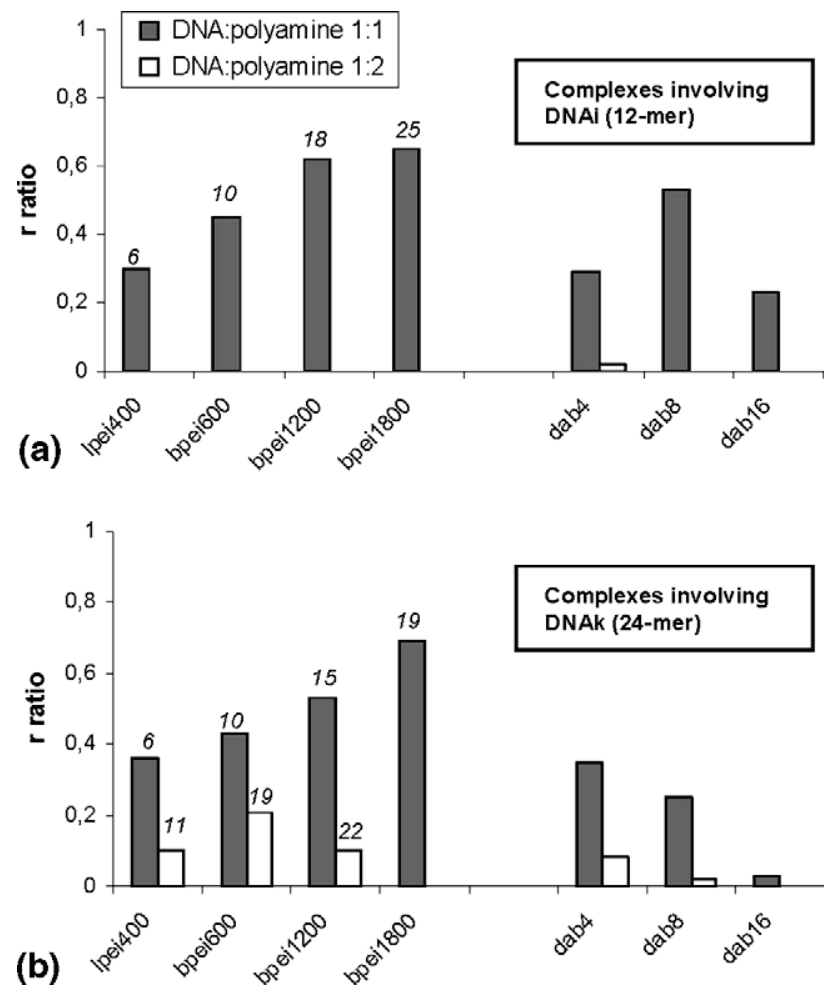

Figure 6. $r$ ratios (of the complex signal over that of the complexed plus the non-complexed DNA) from MALDI-TOF spectra (positive ion mode) of DNAi (a) and DNAk (b), in the presence of four polyethylenimines (LPEI400, BPEI600, BPEI1200, BPEI1800) and three propylenimine dendrimers (DAB4, DAB8, DAB16). For PEI, DP ${ }_{n}$ of bound polymer are indicated in italic on the top of the bars. Relative concentrations [DNA:polybasic compound] were [1:1]. [Ammonium acetate] $=100 \mathrm{mM}$. Matrix: DHAP/ammonium citrate. 


\section{Effect of Polybasic Compound Nature and Size}

The histogram on Figure 6a displays abundance of complexes involving one DNAi and each polyamine listed in Table 1. Data were obtained from several spectra recorded in the positive ion mode. In all cases, relatively intense (1:1) complex was observed and a very small amount of (1:2) complex was sometimes detected; $r$ ratios were always slightly inferior in negative ion mode (data not shown). However, relative intensities of complexes compared to each other were similar in both modes.

As it was seen with BPEI1200, the $\mathrm{DP}_{n}$ of other polyethylenimines bound to DNA were very close in both negative and positive ion modes and relatively close to the values measured by ESI with the polyethylene alone (LPEI400: $\mathrm{DP}_{n \text { pos }}=\mathrm{DP}_{n \text { neg }} \sim 6, \mathrm{DP}_{n \text { ESI }} \sim$ 7; BPEI600: $\mathrm{DP}_{n \text { pos }}=\mathrm{DP}_{n}$ neg $\sim 10, \mathrm{DP}_{n \text { ESI }} \sim 12$; BPEI1800: $\left.\mathrm{DP}_{n \text { pos }} \sim 25, \mathrm{DP}_{n \text { neg }} \sim 23, \mathrm{DP}_{n \text { ESI }} \sim 26\right)$. In the same manner, $\mathrm{I}_{\mathrm{p}}$ of LPEI400, BPEI600, and BPEI1800 were identical in both modes $(1.03,1.06$, and 1.07, respectively) and close to the values measured by ESI (1.04, 1.12, and 1.14, respectively).

For complexes involving polyethylenimines, it was observed that the larger the polyamine, the more abundant the complex (in both negative and positive ion modes). This is consistent with the expected in-solution behavior (the longer the polybasic compound, the more numerous the binding sites, the stronger the interaction). However, the observed trends can reflect differences in desorption/ionization efficiency or gas-phase stability of the complexes, hence no definitive conclusion can be drawn.

A small amount of (DNAi + DAB4) was observed by mass spectrometry despite the weakness of the interaction found by ITC measurements. As previously discussed, (DNAi + DAB8) was observed with a proportion nearly corresponding to that of the solution. The interpretation of relative intensities of (DNAi + DAB16) was less obvious: it is inferior to that of (DNAi + DAB4). This behavior can be due to precipitation phenomenons, the same that prevented to make a $K_{D}$ determination by ITC. It can also be due to the dendrimeric structure of DAB16. Because the positive charges are contained in a limited volume and that some of them can be embedded in the dendrimer inside part, the number of possible bonds binding DNAi to the DAB16 may be too small to permit the complex to survive the MALDI process.

\section{Effect of DNA Composition and Size}

To probe the effect of the DNA base sequence, we recorded MALDI-MS spectra of mixtures involving polybasic compounds and DNAj. DNAj $\left(\mathrm{T}_{4} \mathrm{G}_{6} \mathrm{C}_{2}\right)$ is the complementary strand of DNAi $\left(\mathrm{A}_{4} \mathrm{G}_{2} \mathrm{C}_{6}\right)$. Spectra from DNAj alone (Figure 7a) and from mixtures including K5 (Figure 7b), DAB8 (Figure 7c), and BPEI1200 (Figure 7d) are displayed as examples. Observed species were
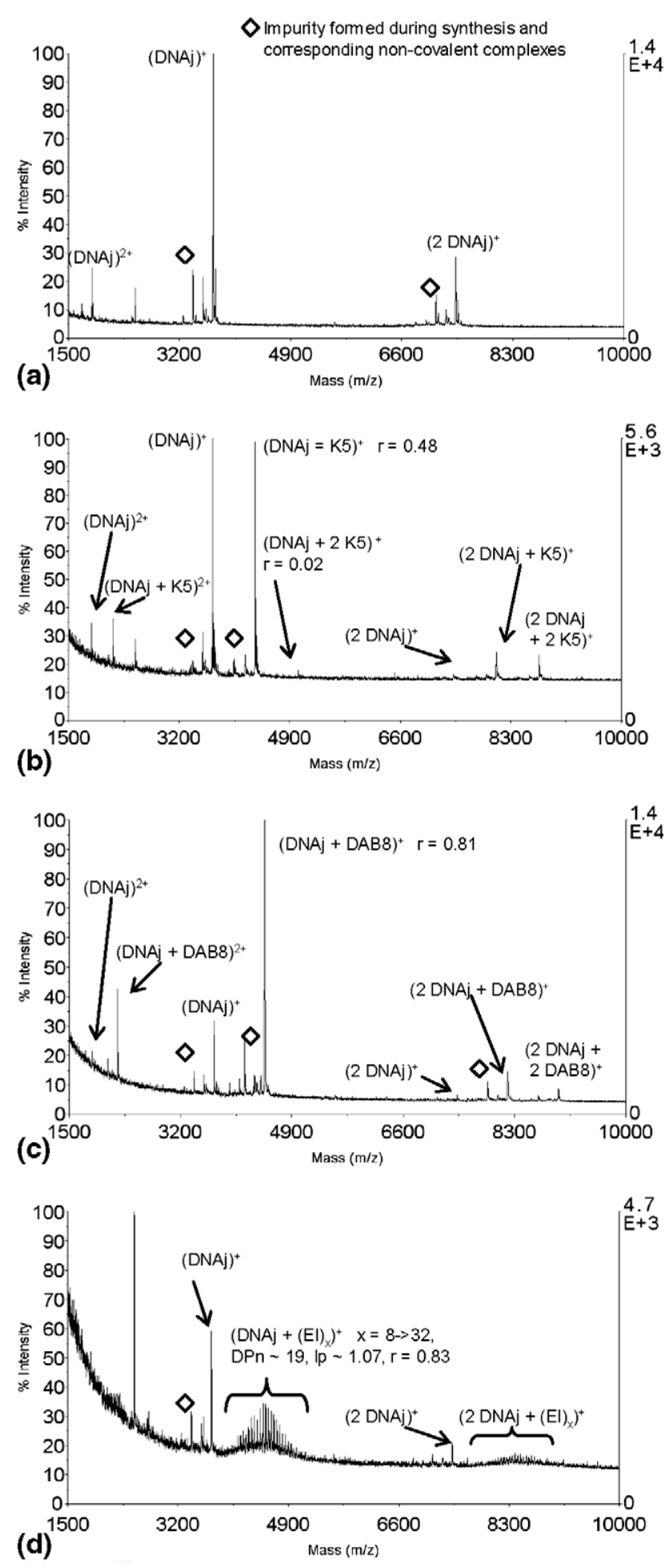

Figure 7. Positive ion mode MALDI-TOF spectra of DNAj alone (a) and in the presence of K5 (b), DAB8 (c), BPEI1200 (d). [Ammonium acetate] $=100 \mathrm{mM}$. Matrix: $\mathrm{DHAP} / \mathrm{ammonium}$ citrate. All charges are the result of deprotonation(s) or protonation(s).

qualitatively the same as in the case of $\mathrm{DNAi} ; r$ ratios were up to 2.5 -fold higher with DNAj than with DNAi (compare Figure $7 b, c$, and d with Figure 2f, g, and h). This was globally confirmed for other polyamines in 
both negative and positive ion modes (not shown), except for the longest PEI for which spectra are difficult to obtain and poorly reproducible. Even if differences of in-solution complexation efficiencies can exist between the two strands, the fact that the complex ions with DNA $j$ are more abundant is likely to be due to response factor or gas-phase stability differences. Indeed, MALDI-MS spectrum of an equimolar mixture of DNAi and DNAj showed a much more intense DNAi peak than the DNAj peak (not shown).

The effect of DNA size was investigated by studying complexes involving DNAk, a 24-mer oligonucleotide with the same proportion of each kind of nucleotidic unit than the 12-mer DNAi. Histogram on Figure 6b displays relative intensities of complexes involving each PEI and DAB. As examples, Figure 8a, b, c, and d are spectra recorded from DNAk alone and from mixtures of DNAk with K5, DAB8, and BPEI1200. When comparing with complexes involving DNAi (Figure 2f, $\mathrm{g}$, and $\mathrm{h}$ ), the disappearance of DNA dimer and its complexes was observed in all cases. This can be due to mass discrimination: the higher masses are generally detected with lower efficiency. In the cases of penta-Llysine and PEIs, complexes were globally more intense when involving the 24-mer DNA than when involving the 12-mer DNA. As seen previously, this difference can be due to a greater stability of the complexes or to a lesser desorption/ionization efficiency of the longer free DNA. Like the complexes involving the 12-mer DNA, Figure $6 \mathrm{~b}$ shows that the larger the PEI, the more intense the complex. On the other hand, it was observed that the larger the $\mathrm{DAB}$, the less abundant the complex compared with DNA alone. Complexes involving DAB8 and DAB16 were less intense with DNAk than with DNAi. This behavior seems to confirm that largest (DNA + DAB) complexes would not have a sufficient number of bonds to be stable in the gas phase.

It is noteworthy that while the $\mathrm{DP}_{n}$ of LPEI400 and BPEI600 bound to DNA were similar to those observed with DNAi, the DP ${ }_{n}$ of bound BPEI1200 and BPEI1800 are lower than with 12-mer DNA, maybe due to high mass discrimination.

When comparing complexes involving DNAk with those involving DNAi, the presence of significant (1:2) complexes was only observed in the former case. These complexes were intense for K5, LPEI400, BPEI600, BPEI1200, and DAB4. In the case of (DNAk + LPEI400) and (DNAk + BPEI600), the (1:2) complexes involved polymers with an average $\mathrm{DP}_{n}$ nearly equal to that of the polymers present in (1:1) complexes. In the case of (DNAk + BPEI1200), polymers involved in (1:2) complexes were shorter than those involved in the (1:1) complex.

To determine the maximum stoichiometry according to both the length of the DNA and the type and length of the polybasic compound, spectra were recorded with a 10-fold excess of polybasic compound. Results are reported in Figure 9a and b. For (DNAk + BPEI1200) and (DNAk + BPEI1800), signals were not good enough
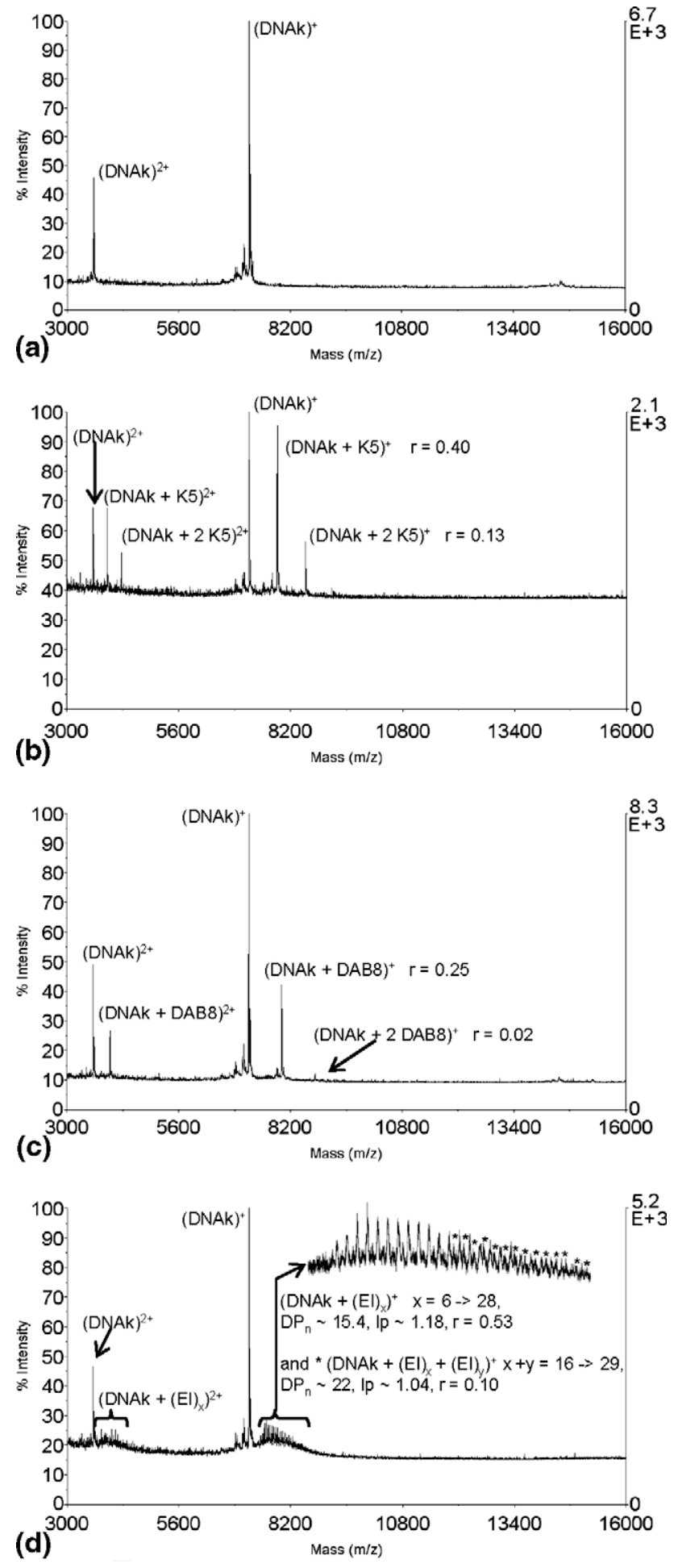

Figure 8. Positive ion mode MALDI-TOF spectra of DNAk alone (a) and in the presence of K5 (b), DAB8 (c), BPEI1200 (d). [Ammonium acetate] $=100 \mathrm{mM}$. Matrix: DHAP/ammonium citrate. All charges are the result of deprotonation(s) or protonation(s).

to differentiate (1:1) and (1:2) complexes. When DNAi (12-mer) was involved, no significant (1:2) complex was detected (Figure 9a). Even the smallest polybasic compounds (LPEI400, $\sim 7$ amino groups and DAB 4,6 


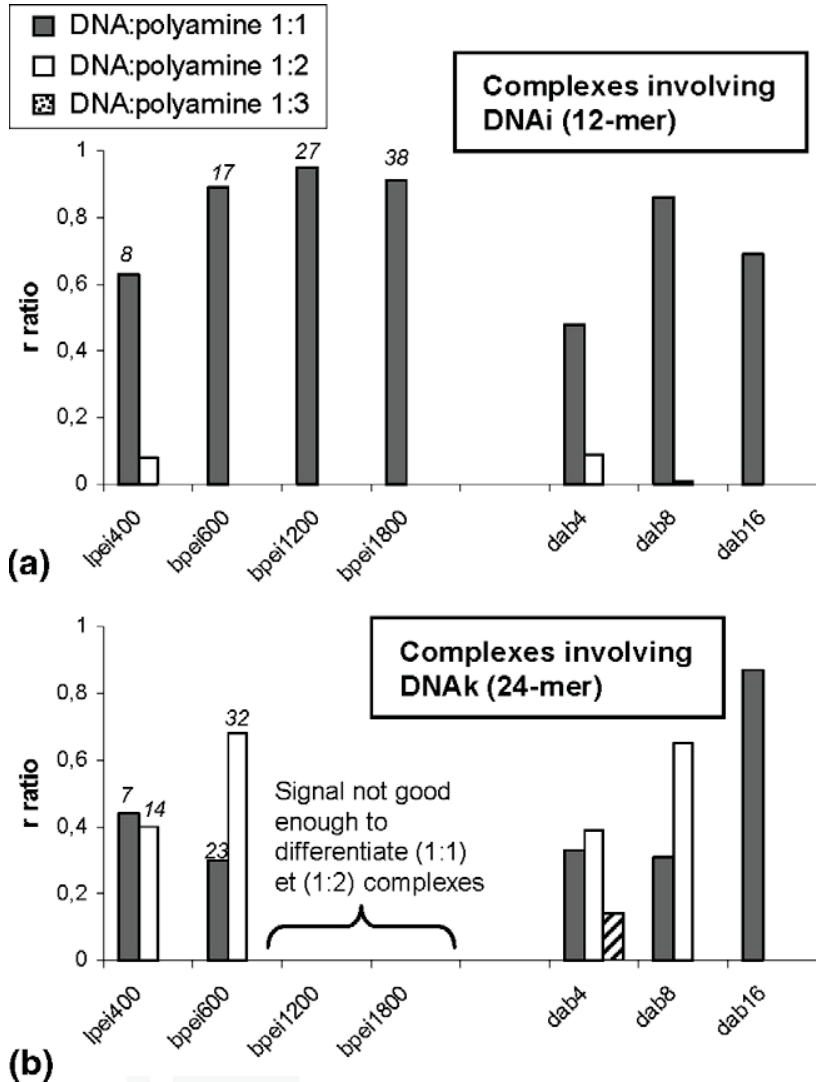

Figure 9. $\quad r$ ratios in the positive ion mode MALDI-TOF spectra of DNAi (a) and DNAk (b), each with four polyethylenimines (LPEI400, BPEI600, BPEI1200, BPEI1800) and three propylenimine dendrimers (DAB4, DAB8, DAB16). For PEI, $\mathrm{DP}_{n}$ of bound polymer are indicated in italic. Relative concentrations [DNA:polybasic compound] were [1:10]. [Ammonium acetate] $=100 \mathrm{mM}$. Matrix: DHAP/ammonium citrate.

amino groups) mainly bound to DNA (11 phosphate groups) with a (1:1) stoichiometry. On the other hand, several complexes involving DNAk were detected under their (1:2) and sometimes (1:3) forms (Figure 9b). The presence of these (1:2) and (1:3) stoichiometries for the 24-mer oligonucleotide shows, as expected, that a 24-mer DNA has potentially more available contact points than a 12-mer. In contrast, a (1:1) complex with 12-mer DNAi would carry an insufficient number of negative charges to efficiently bind to a second polyamine. The need for a large number of bonds to form a stable association could also explain why $\mathrm{DP}_{n}$ of BPEI1200 involved in (1:2) complex is smaller than that involved in (1:1) complex. Lastly, to bind to the maximum number of basic sites of a polybasic compound, single-stranded DNA may have to adopt a folded conformation, making free phosphate groups unavailable for a second association.

Surprisingly, $\mathrm{DP}_{n}$ of PEI bound to DNA were found to be clearly higher when PEI is present in excess in the solution (compare Figure 6 with Figure 9). Values measured in excess of polybasic compound are relatively close to the manufacturer's values. We expected the larger chains to be bound more efficiently than the shorter ones. Actually, their association was observed only when forced by a molar excess of polyamine.

\section{Effect of Matrix and Salt Concentration}

Spectra of DNAi + polybasic compound mixtures were recorded with ATT and DHAP as matrixes with different ammonium acetate concentrations. When increasing ammonium acetate concentration (with DHAP as the matrix), $r$ ratio globally decreased for complexes involving K5 (Figure 10a), PEIs and DABs (data not shown). By assuming that the response factors of the alone DNAi and its complexes are affected in the same ratio by the increase of the ammonium concentration, this can be interpreted as a decrease in complex formation. This result was expected since ammonium ions from the buffer can compete in solution with protonated binding sites of polybasic compounds to bind to DNA phosphate groups, and thus reduce the affinity through electrostatic interactions between the two components [31].

When ATT was used as the matrix, no complex was detected between DNAi and polyamines. In the case of (DNAi + K5), a small amount of (1:1) complex was observed only for low ammonium acetate concentration. Interestingly, the relative abundance of (DNAi + R5) remained constant whatever the matrix and the
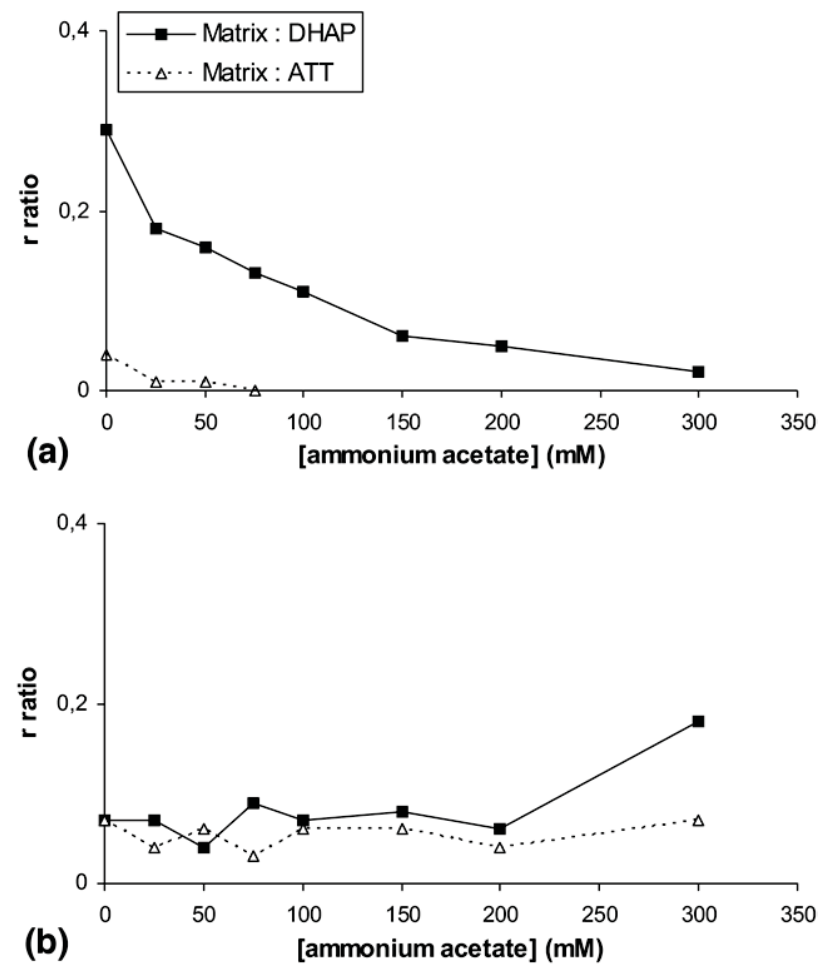

Figure 10. $r$ ratios in the negative ion mode MALDI-TOF spectra of DNAi in the presence of K5 (a) and R5 (b). Relative abundances are reported as a function of the concentration of ammonium acetate with both DHAP/ammonium citrate and ATT/ammonium citrate as matrixes. 


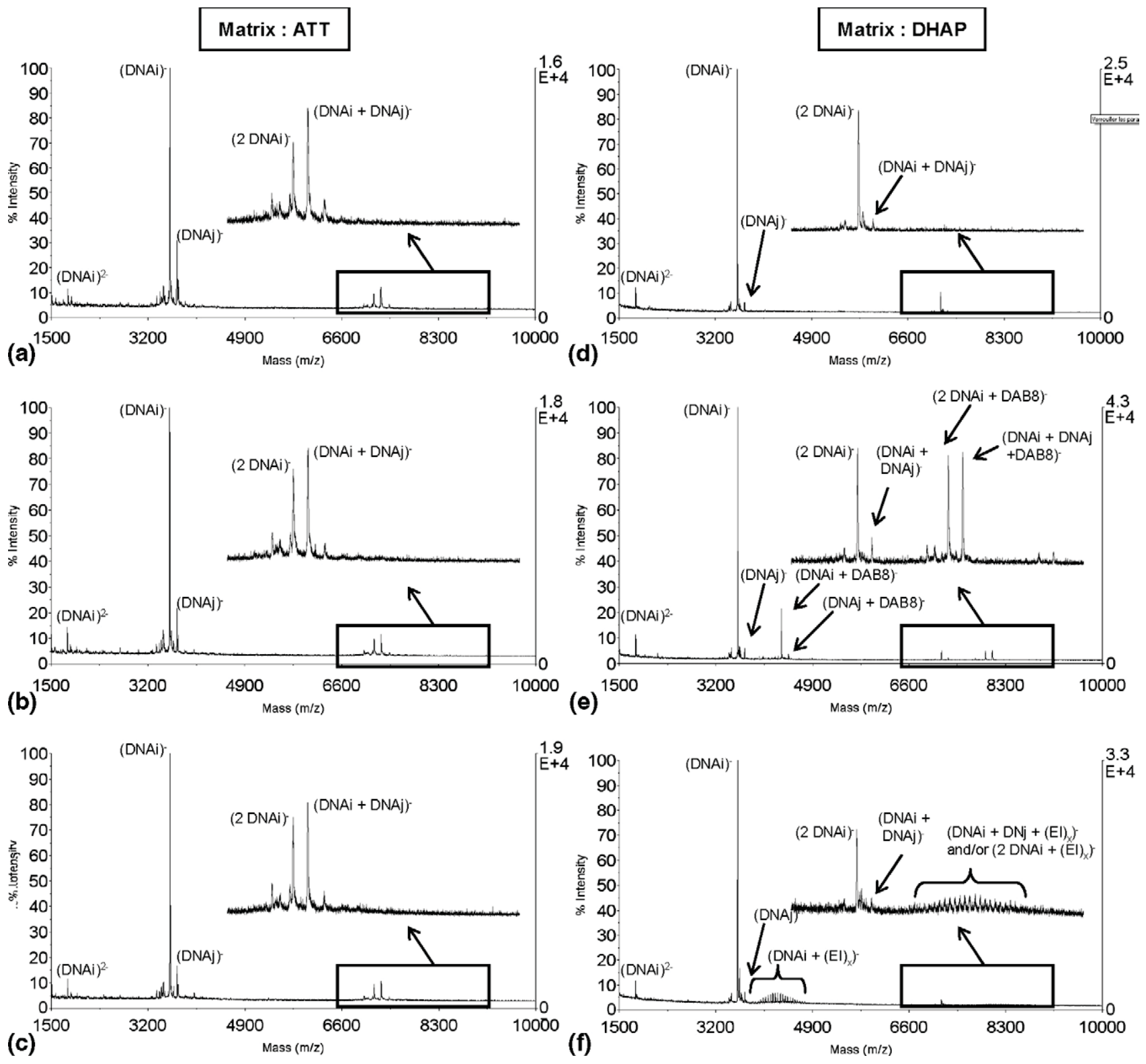

Figure 11. Negative ion mode MALDI-TOF spectra of an annealed mixture of DNAi and DNAj alone (a) and (d) and in the presence of DAB8 (b) and (e) and BPEI1200 (c) and (f). [Ammonium acetate] = $100 \mathrm{mM}$. (a), (b), and (c): The matrix is ATT/ammonium citrate. (d), (e), and (f): The matrix is DHAP

/ ammonium citrate. All charges are the result of deprotonation(s) or protonation(s).

ammonium acetate concentration (from 0 to $300 \mathrm{mM}$ ). These experiments emphasize the need for the use of a specific matrix according to the nature of the polybasic compound.

\section{Observation of Complexes Involving Duplex DNA and Polybasic Compound}

In the course of our study, DHAP was found to favor the observation of (single-stranded DNA + polybasic compound) complexes better than ATT (details not shown). However, ATT is a well-known matrix used to observe DNA specific duplex [19, 32]. Consequently, both DHAP and ATT were used to study complexes between DNA duplex and polybasic compounds. In Figure 11, the spectra of duplex alone, (duplex + DAB8) and (duplex + PEI1200) are displayed as examples. Figure 11a shows that ATT allowed the observation of specific duplex (DNAi + DNAj). Non-specific (two DNAi) dimer was detected too with a similar intensity. However, since the detection of DNAi is much better than that of DNAj (as seen previously), we can expect a poor detection of the specific duplex compared to nonspecific DNAi dimer. As a consequence, even if gas-phase aggregation is likely to occur, specific dimer seems to be favoured over nonspecific dimer. As seen on Figures $11 \mathrm{~b}$ and c, absolutely no (DNA + polybasic compound) complex was detected with ATT. In con- 
trast, when DHAP was used as matrix (Figures 11d, e, and $\mathrm{f}$ ), nonspecific dimer was clearly more abundant than specific duplex. Complexes involving one DNA and one polybasic compound were observed with DAB8 and BPEI1200. Moreover, complexes involving a nonspecific dimer and/or a specific duplex were observed. While the complex involving DAB8 and the nonspecific DNAi dimer is of similar abundance than the free dimer, that involving the specific duplex is 6-fold higher. This could indicate that complexes involving specific duplex are favoured over those involving nonspecific dimer of DNA. This can be explained by the fact that at least a part of the specific complex pre-exists in the MALDI deposit, while the nonspecific one is likely formed in the gas phase. The quality of the spectrum of (DNAi + DNAj + BPEI1200) did not permit the differentiation of complexes involving specific duplex from those involving nonspecific dimer. The case of these tertiary complexes are more complicated than that of the (single-stranded DNA + polybasic compound) complexes. ATT seems to be the most appropriate matrix for the detection of specific duplex held by hydrogen bonding and $\pi$-stacking, while only DHAP allows the detection of complexes held by ionic interactions. To date, the reasons explaining the differences of behavior between these two matrixes are not clearly understood.

\section{Conclusions}

MALDI-TOF MS was successfully used to detect noncovalent complexes between DNA and various polybasic compounds. The originality of this work lies in the fact that the noncovalent complexes included polydisperse polymers as basic compounds. Moreover, noncovalent complex studies using MALDI as ionization technique are somewhat unusual. Control experiments showed that detected complexes by mass spectrometry were not the result of gas-phase aggregation but rather were pre-existing in matrix crystals and that the species survived the ionization process at least in part. It was also shown that the noncovalent complexes were mainly the result of electrostatic interactions as expected. From the MS data, differences in relative abundances and stoichiometries were found to be related to the nature and the size of the binding partners (DNA, polybasic compound). Most of the findings deduced from MS data were consistent with the known or the expected in solution behavior. The same complexes were detected both in the negative and positive ion mode. Sample preparation before MALDI experiments was found to be crucial. In particular, the choice of the matrix determines the observation of the complexes according to the nature of the involved polybasic compound and the ammonium acetate concentration. The main limitation of MALDI-TOF detection is that the abundance of the observed species depends on their desorption/ionization efficiencies. An addi- tional difficulty arose when studying complexes involving a DNA duplex and a polybasic compound. Indeed, the most appropriate matrix for the observation of DNA duplex (ATT) was not the most appropriate for the observation of DNA/polyamine complex (DHAP). However, speed and ease of use of MALDI-TOF MS support its use for this kind of study. In a previous ESI-MS report [7], it has been shown that Coulomb repulsions could have an influence on the stability of the multiply charged complexes. The production of singly charged species might be another important advantage of MALDI over the electrospray technique. MALDI-MS/MS experiments are in the process of being carried out and we hope this could bring important complementary information.

\section{Acknowledgments}

Microcalimetry experiments were conducted by MIP laboratory in Orsay, France. The authors gratefully acknowledge financial support from the Association Française contre les Myopathies (AFM).

\section{References}

1. Chirila, T. V.; Rakoczy, P. E.; Garrett, K. L.; Lou, X.; Constable, I. J. The Use of Synthetic Polymers for Delivery of Therapeutic Antisense Oligodeoxynucleotides. Biomaterials 2002, 23, 321-342.

2. Zhang, S.; Xu, Y.; Wang, B.; Qiao, W.; Liu, D.; Li, Z. Cationic Compounds Used in Lipoplexes and Polyplexes for Gene Delivery. J. Controlled Release 2004, 100, 165-180.

3. Yamashita, M.; Fenn, J. B. Electrospray Ion Source. Another Variation on the Free-Jet Theme. J. Phys. Chem. 1984, 88, 4451-4459.

4. Tanaka, K.; Waki, H.; Ido, Y.; Akita, S.; Yoshida, T. Protein and Polymer Analyses up to $m / z 100,000$ by Laser Ionization Time-of-flight Mass Spectrometry. Rapid Commun. Mass Spectrom. 1988, 2, 151-153.

5. Karas, M.; Hillenkamp, F. Laser Desorption Ionization of Proteins with Molecular Masses Exceeding 10,000 Daltons. Anal. Chem. 1988, 60, 2299-2301.

6. Loo, J. A. Studying Noncovalent Protein Complexes by Electrospray Ionization Mass Spectrometry. Mass Spectrom. Rev. 1997, 16, 1-23.

7. Terrier, P.; Tortajada, J.; Buchmann, W. A Study of Noncovalent Complexes Involving Single-Stranded DNA and Polybasic Compounds Using Nanospray Mass Spectrometry. J. Am. Soc. Mass Spectrom. 2007, $18,346-358$.

8. Woods, A. S.; Ferré, S. Amazing Stability of the Arginine-Phosphate Electrostatic Interaction. I. Proteome Res. 2005, 4, 1397-1402.

9. Jackson, S. N.; Wang, H.-Y. J.; Woods, A. S. Study of the Fragmentation Patterns of the Phosphate-Arginine Noncovalent Bond. J. Proteome Res. 2005, 4, 2360-2363.

10. Jackson, S.; N.; Wang, H.-Y. J.; Yergey, A.; Woods, A. S. Phosphate Stabilization of Intermolecular Interactions. J. Proteome Res. 2006, 5, 122-126

11. Sudha, R.; Kohtani, M.; Jarrold, M. F. Noncovalent Interactions Between Unsolvated Peptides: Helical Complexes Based on Acid-Base Interactions. J. Phys. Chem. B 2005, 109, 6442-6447.

12. Bolbach, G.; Matrix-Assisted Laser. Desorption/Ionization Analysis of Noncovalent Complexes: Fundamentals and Applications. Curr. Pharm. Des. 2005, 11, 2535-2557.

13. Juhasz, P.; Biemann, K.; Mass Spectrometric. Molecular-Weight Determination of Highly Acidic Compounds of Biological Significance via their Complexes with Basic Polypeptides. Proc. Natl. Acad. Sci. U.S.A. 1994, 91, 4333-4337.

14. Tang, X.; Callahan, J. H.; Zhou, P.; Vertes, A. Noncovalent ProteinOligonucleotide Interactions. Monitored by Matrix-Assisted Laser Desorption/Ionization Mass Spectrometry. Anal. Chem. 1995, 67, 45424548.

15. Lin, S.; Cotter, R. J.; Woods, A. S. Detection of Noncovalent Interaction of Single and Double Stranded DNA With Peptides by MALDI-TOF. Prot. Struct. Funct. Genet. Suppl. 1998, 2, 12-21.

16. Ohara, K.; Smietana, M.; Vasseur, J.-J. Characterization of Specific Noncovalent Complexes between Guanidinium Derivatives and SingleStranded DNA by MALDI. J. Am. Soc. Mass Spectrom. 2006, 17, 283-291.

17. Lin, S.; Long, S.; Ramirez, S. M.; Cotter, R. J.; Woods, A. S. Characterization of the "Helix Clamp" Motif of HIV-1 Reverse Transcriptase Using MALDI-TOF MS and Surface Plasmon Resonance. Anal. Chem. 2000, 72, 2635-2640. 
18. Luo, S.-Z.; Li, Y.-M.; Qiang, W.; Zhao, Y.-F.; Abe, H.; Nemoto, T.; Qin, X.-R.; Nakanishi, H. Detection of Specific Noncovalent Interaction of Peptide with DNA by MALDI-TOF. J. Am. Soc. Mass Spectrom. 2004, 15, $28-31$

19. Lecchi, P.; Pannell, L. K. The Detection of Intact Double-Stranded DNA by MALDI. J. Am. Soc. Mass Spectrom. 1995, 6, 972-975.

20. Woods, A. S.; Huestis, M. A. A Study of Peptide-Peptide Interaction by Matrix-assisted Laser Desorption/Ionization. J. Am. Soc. Mass Spectrom. 2001, 12, 88-96.

21. Ciruela, F.; Burgueño, J.; Casado, V.; Canals, M.; Marcellino, D.; Goldberg, S. R.; Bader, M.; Fuxe, K.; Agnati, L. F.; Lluis, C.; Franco, R.; Ferré, S.; Woods, A. S. Combining Mass Spectrometry and Pull-Down Techniques for the Study of Receptor Heteromerization. Direct EpitopeEpitope Electrostatic Interactions Between Adenosine $\mathrm{A}_{2 \mathrm{~A}}$ and Dopamine $\mathrm{D}_{2}$ Receptors. Anal. Chem. 2004, 76, 5354-5363.

22. Woods, A. S. The Mighty Arginine, the Stable Quaternary Amines, the Powerful Aromatics, and the Aggressive Phosphate: Their Role in the Noncovalent Minuet. J. Proteome Res. 2004, 3, 478-484.

23. Zehl, M.; Allmaier, G. Investigation of Sample Preparation and Instrumental Parameters in the Matrix-Assisted Laser Desorption/Ionization Time-of-Flight Mass Spectrometry of Noncovalent Peptide/Peptide Complexes. Rapid Commun. Mass Spectrom. 2003, 17, 1931-1940.

24. Asara, J. M.; Allison, J. Enhanced Detection of Oligonucleotides in UV MALDI MS Using the Tetra-Amine Spermine as a Matrix Additive. Anal. Chem. 1999, 71, 2866-2870.
25. Vandell, V. E.; Limbach, P. A. Polyamine Comatrices for MatrixAssisted Laser Desorption/Ionization Mass Spectrometry of Oligonucleotides. Rapid. Commun. Mass Spectrom. 1999, 13, 2014-2021.

26. Distler, A. M.; Allison, J. 5-Methoxysalicylic Acid and Spermine: A New Matrix for the Matrix-Assisted Laser Desorption/Ionization Mass Spectrometry Analysis of Oligonucleotides. J. Am. Soc. Mass Spectrom. 2001, $12,456-462$.

27. Distler, A. M.; Allison, J. Additives for the Stabilization of DoubleStranded DNA in UV-MALDI MS. J. Am. Soc. Mass Spectrom. 2002, 13, 1129-1137.

28. Bolbach, G. Matrix-Assisted Laser Desorption/Ionization Analysis of Noncovalent Complexes: Fundamentals and Applications. Curr. Pharm. Des. 2005, 11, 2535-2557.

29. Bogan, M. J.; Agnes, G. R. Time-of-Flight Mass Spectrometric Analysis of Ions Produced from Adjacent Sample Spots Irradiated Simultaneously by a Single $337 \mathrm{~nm}$ Laser. Rapid Commun. Mass Spectrom. 2003, $17,2557-2562$.

30. Friess, S. D.; Zenobi, R. Protein Structure Information from Mass Spectrometry? Selective Titration of Arginine Residues by Sulfonates. J. Am. Soc. Mass Spectrom. 2001, 12, 810-818.

31. Bloomfield, V. A.; Crothers, D. M.; Tinoco, J. I. Nucleic Acids, Structures, Properties, and Functions; University Science Books: Sausalito, CA, 1999, pp. 475-534.

32. Sudha, R.; Zenobi, R. The Detection and Stability of DNA Duplexes Probed by MALDI Mass Spectrometry. Helv. Chim. Acta 2002, 85, 3136-3143. 